\title{
Functional cerebral changes in multiple sclerosis patients during an autobiographical memory test
}

\author{
Alexandra Ernst ${ }^{1,2}$, Vincent Noblet ${ }^{2,3,4}$, Ekaterina Denkova ${ }^{2}$, Frederic Blanc ${ }^{2,3,4,5}$, Jérôme de Seze ${ }^{2,5,6}$, Daniel \\ Gounot $^{2,3}$, and Liliann Manning ${ }^{1,2}$ \\ ${ }^{1}$ Cognitive Neuropsychology and Physiopathology of Schizophrenia (INSERM UMR 1114), Strasbourg, France \\ ${ }^{2}$ Department of Psychology, University of Strasbourg, Strasbourg, France \\ ${ }^{3}$ ICube (CNRS UMR 7357), Strasbourg, France \\ ${ }^{4}$ Fédération de Médecine translationnelle de Strasbourg (FMTS), Strasbourg, France \\ ${ }^{5}$ Department of Neurology and Centre Mémoire de Ressources et de Recherche (CMRR), University Hospital of \\ Strasbourg, Strasbourg, France \\ ${ }^{6}$ Clinical Investigation Centre (CIC, INSERM 1434), University Hospital of Strasbourg, Strasbourg, France
}

\begin{abstract}
Our aim was to investigate the functional underpinnings of autobiographical memory (AM) impairment in multiple sclerosis (MS) patients. To that end, 18 patients and 18 controls underwent the autobiographical interview (AI). Subsequently, the 36 participants underwent a functional magnetic resonance imaging (fMRI) session designed to assess the construction and elaboration of AMs. A categorical control task was also presented. Patients were trained in the fMRI procedure to optimise the procedural aspects accompanying the task itself. Although the patients obtained significantly poorer AI scores $(p<.001)$, their performance on the easier AM fMRI task was efficiently carried out, allowing relevant comparisons with healthy controls. Relatively to healthy controls, the patients showed increased and bilateral cerebral activations $(p<.005)$ during the construction and elaboration phases. The prefrontal, temporal and posterior cerebral region activations were located within the core network sustaining AM, with the bilateral prefrontal region being centrally involved. The parametric neural responses to the difficulty of access and amount of details of memories were also significantly different for the two groups, with the right hippocampal region showing a particularly increased recruitment $(p<$ $.005)$. The findings suggested the presence of functional cerebral changes during AM performance and supported the presence of AM retrieval deficit in MS patients.
\end{abstract}

Keywords: Cerebral activation changes; Multiple sclerosis; Autobiographical memory; Autobiographical interview; Functional MRI.

\section{Acknowledgments}

We thank C. Vinet-Gasse and A. Botzung for helping with testing and V. Voltzenlogel for interrater reliability scoring, N. Heider, S. Graves, F. Ernwein, A. Clerc-Renault, E. Montaut and the NCC MA students for the transcriptions of the AI audio-recordings. We are grateful to the "Imagerie in vivo" Platform (ICube laboratory), where the MRI examination was realised, the CIC of the Strasbourg University Hospitals, where patients and controls were examined, C. Marrer for the fMRI technical assistance and O. Després for initial participation in E Prime programming.

We are grateful to the "Fondation pour la Recherche sur la Sclérose en Plaques" (ARSEP; Ile de France; grant to L.M) for research funding and to the Ministry of National Education and Research (A.E.'s Ph.D. grant). 
The occurrence of functional cerebral activation changes in neurological patients is a well-established phenomenon, nowadays (Chen, Epstein, \& Stern, 2010). Functional cerebral changes have been described in different conditions such as multiple sclerosis (MS; Filippi \& Rocca, 2004), Alzheimer's disease (Bookheimer et al., 2000), stroke (Johansson, 2000) or traumatic brain injury (Levin, 2003), and in various cognitive domains. However, only a few investigations explored the functional underpinnings of autobiographical memory (AM). Briefly stated, AM is the ability to mentally re-experience personal past events, within a specific spatio-temporal context, as they are remembered (Tulving, 2002). Regarding the literature, AM impairment has been frequently reported and well described at the clinical level in various clinical conditions, for instance in Alzheimer's disease patients (Addis, Sacchetti, Ally, Budson, \& Schacter, 2009), schizophrenic patients (D'Argembeau, Raffard, \& Van Der Linden, 2008), depressed persons (Williams et al., 1996), patients with medial temporal lobe amnesia (Has-sabis, Kumaran, Vann, \& Maguire, 2007) or patients with fronto-temporal dementia (Irish, Hodges, \& Piguet, 2013). In contrast, according to Maguire, Vargha Khadem, and Mishkin (2001), the dearth of clinical AM neuroimaging studies is probably related to the difficulty to interpret the neuroimaging findings when patients cannot perform the AM task with sufficient accuracy and reaction times equivalent to those of healthy subjects. Nonetheless, by controlling these methodological constraints, some studies provide relevant insights into the influence of brain pathology on the AM neural bases. For instance, cerebral activation changes relatively to healthy controls have been shown in bilateral hippocam-pal atrophy (Maguire et al., 2001), amnestic mild cognitive impairment (Poettrich et al., 2009), Alzheimer's disease (Meulenbroek, Rijpkema, Kessels, Olde Rikkert, Fernandez, 2010) or in a semantic dementia case study (Maguire, Kumaran, Hassabis, \& Kopelman, 2010).

Recently, using a stringent assessment test, AM has been shown to be impaired in MS patients (Ernst et al., 2012, 2013, 2014). These studies show that AM impairment in MS patients is manifested by difficulties to both initially retrieve personal memories and recollect details associated with personal events. Specifically, Ernst and co-workers suggested a retrieval deficit hypothesis, related to a main prefrontal dysfunction, to explain AM impairment in MS patients. More generally, the multifocal nature of MS brain lesions (e.g., Trapp \& Nave, 2008), together with the widespread neural network sustaining AM, have been suggested to account for AM impairment in MS patients. More precisely, on the one hand, AM processes have been primarily associated with a brain network including the medial prefrontal cortex, the medial and lateral temporal cortices, the parietooccipital regions and the temporo-parietal junction (Svoboda, McKinnon, \& Levine, 2006). On the other hand, several studies carried out in MS patients have demonstrated that structures such as the frontal cortex (Benedict et al., 2002; Bendfeldt et al., 2009) and the hippocampus (Sicotte et al., 2008; Hulst et al., 2012), which are essential in the AM core network, are frequently damaged. In parallel, previous neuroimaging studies conducted in MS patients have reported cerebral activation changes, entailing increased activation in brain areas also recruited by healthy controls and/or the recruitment of alternative brain areas (Hulst et al., 2012), during the completion of attention and working memory (Kollndorfer et al., 2013), ante-rograde memory (Hulst et al., 2012; Mainero et al., 2004; Morgen et al., 2007) or executive functions (Lazeron, Rombouts, Scheltens, Polman, \& Barkhof, 2004) tasks. In this context, the occurrence of functional activation changes could be expected in the context of AM impairment in MS patients. However, to our knowledge, only one preliminary study (Ernst et al., 2012) addressed this question, showing cerebral activation changes, mainly located in the bilateral prefrontal regions, during the retrieval of personal memories in MS patients with impaired AM performance.

Thus, based on these theoretical framework and preliminary findings, the aim of the present study was to investigate more extensively the neural bases of AM impairment in MS patients. Bearing in mind that AM plays a major role in daily life functioning and is ultimately linked to the self (Rasmussen \& Habermas, 2011), its impairment can lead to debilitating consequences, which emphasises the clinical necessity of this kind of investigations. To that end, we studied (1) the functional cerebral substrates sustaining both the access and further recollection of details associated with personal memories and (2) the influence of recollective qualities of memories on cerebral activations in MS patients. Following Ernst et al., (2012), we hypothesised that functional activation changes would be predominantly observed in the prefrontal regions during retrieval and elaboration of memories. Moreover, considering the evidence of hippocampal damage in MS patients (Hulst et al., 2012; Sicotte et al., 2008), the role of the hippocampus in AM, and evidence suggesting that the hippocampal activity is modulated by the recollective qualities of memory (Addis, Moscovitch, Crawley, \& McAndrews, 2004; Addis \& Schacter, 2008), we also expected functional changes in the hippocampal region in MS patients, relatively to healthy controls.

\section{MATERIALS AND METHODS}

\section{Subjects}

Eighteen patients with definite MS according to the McDonald criteria (McDonald et al., 2001) were recruited at 
the Neurology Units of two French hospitals (Strasbourg and Colmar). Inclusion criteria were the diagnosis of a relapsing-remitting disease course, Expanded Disability Status Scale (EDSS; Kurtzke, 1983) score $\leq 5$, absence of major signs of depression according to the Montgomery and Asberg Depression Rating Scale (Montgomery \& Asberg, 1979; clinical threshold score $\geq 15$ ), no recent exacerbation of MS symptoms and AM impairment (see below). Eighteen healthy controls matched for gender, age, education level and verbal intellectual quotient (IQ) were recruited. Exclusion criteria for all the subjects were documented psychiatric illness, neurological disorder (other than MS for the patients) and poor knowledge of French. Demographic and clinical data are summarised in Table 1. The present study was approved by the "Committee for Protection of Persons" (CPP/ CNRS ${ }^{\circ}$ 07023) and we complied with the Declaration of Helsinki.

\section{Behavioural assessment}

In the first step, all the patients underwent a neuropsychological baseline examination covering the following cognitive domains: the general verbal abilities were tested with the Wechsler Adult Intelligence Scale-Ill (Wechsler, 1997) verbal IQ, short form (Axelrod, Ryan, \& Ward, 2001). Non-verbal reasoning was assessed using the Advanced Progressive Matrices Set 1 (Raven, 1958). Anterograde memory was examined with the Rey auditory verbal learning test (Rey, 1964), and the Rey-Osterrieth Complex Figure (Rey, 1941; Osterrieth, 1944). The executive functions were probed by means of the phonological and categorical fluency tests (National Hospital, London), the Brixton Spatial Anticipation test (Burgess \& Shallice, 1997), the Tower of London (Shallice, 1982) and the Cognitive Estimation Task (Shallice \& Evans, 1978). The attentional abilities and information processing were assessed using the Information Processing Speed test from the Adult Memory Information Processing Battery (Coughlan \& Hollows, 1985), the Stroop test (Stroop, 1935) and the months backward test (National Hospital, London). Language was tested with the Déno 100 test (Kremin, 2002), and the visuoperceptual and visuospatial abilities with the Silhouettes and Cube Analysis sub-tests from the Visual Object and Space Perception Battery (VOSP; Warrington \& James, 1991). Additionally, the effect of fatigue in everyday life was assessed using the "Echelle de Mesure de l'Impact de la Fatigue" (Debouverie, PittionVouyovitch, Louis, \& Guillemin, 2007).

The AM assessment was carried out using the autobiographical interview (AI), kindly communicated by B. Levine to LM (Levine, Svoboda, Hay, Winocur, \& Moscovitch, 2002) for the patients and the healthy controls. The AI consists in retrieving personal unique events, temporally and contextually specific, occurring over minutes to hours (but no longer than 1 day), and to generate freely as many details as possible about the event. We used an adapted version of the Al, allowing the introduction of cue-words (Addis, Wong, \& Schacter, 2008; Addis et al., 2009) and the possibility to probe a greater number of past recollections to assess more comprehensively the retrieval process (Barnabe, Whitehead, Pilon, Arsenault-Lapierre, \& Chertkow, 2012; Leyhe, Muller, Milian, Eschweiler, \& Saur, 2009). Three past events per life period were collected [i.e., four or five life periods, depending on the subject's age; 0-11 years, 12-20 years, 21 to (current age -1) or 21-35 years, 36 to (current age -1) and the previous year]. Participants were informed that the cue-words were intended to be used flexibly and no time limit was set to avoid the potential influence of the patients' slowed down cognitive processing speed on AM performance. The present work focuses on the free recall phase deficit specifically.

The AI session was audio-recorded for later transcription and scoring following the standardised procedure supplied by Levine et al. (2002).

Since our aim was to explore the functional underpinnings of AM impairment in MS patients, we included patients, whose AM scores were below the mean of the normative data, which was provided for the French version of the AI by Ernst et al. (2012). In this context, the presence of AM impairment was determined by the free recall phase scores, and especially the mean number of internal details, which is an objective measure of the episodic quality of memories. Thus, MS patients' AM performance was deemed to be impaired if the mean score for internal details was $\leq 22.12$

TABLE 1: Demographical and clinical data

\begin{tabular}{llll}
\hline & MS patients & Healthy controls & $\begin{array}{l}\text { Statistical analysis: } \\
\text { Test and } \mathrm{p} \text { values }\end{array}$ \\
\hline$N$ & 18 & 18 & - \\
Age in years & $41.11(10.22)$ & $39.72(8.61)$ & $t=0.44 ; p=.66$ \\
Education in years & $13.22(2.05)$ & $13.44(2.45)$ & $t=-0.29 ; p=.80$ \\
Sex (ratio female/male) & $15 / 3$ & $15 / 3$ & $\mathrm{X}^{2}=0.0 ; p=1.0$ \\
EDSS [range] & $2.50(1.37)[0-5]$ & - & - \\
Duration of MS in years & $12.53(8.68)$ & - & - \\
Number of DMD treatment & $1.0(0.0)$ & - & -
\end{tabular}




Verbal IQ $99.78(11.94) \quad 98.06(12.10) \quad t=0.43 ; p=.67$

Mean (and standard deviation) for the MS patient and the healthy control groups. DMD, disease-modifying drugs.

\section{Neuroimaging data}

\section{Functional magnetic resonance imaging tasks}

Two functional magnetic resonance imaging (fMRI) tasks were proposed during scanning. The experimental task (i.e., past events condition) consisted in the evocation of unique personal past events, contextually specific, occurring over minutes or hours, but no longer than 1 day. Examples were provided and the similarity to the instructions and event's characteristics of the AI was reminded. To guide memory recollection, two cue-words were provided during the past events task (instead of a single cue-word during the AI) and a total of 32 pairs of words were proposed to elicit memories (e.g., "store-crowd"), covering the same life periods that were proposed in the AI [i.e., four or five life periods, depending on the subject's age; 0-11 years, 12-20 years, 21 to (current age -1) or 21-35 years, 36 to (current age -1) and the previous year]. Importantly, during the fMRI task, we used a list of arbitrary cue-words, similar for all the participants. Notwithstanding the fact that the cue-words were different from the cue-words used during the AI, participants were instructed to evoke memories different than those previously recollected during the AI to avoid a "re-encoding effect" of memories (Svoboda et al., 2006). Moreover, they were informed that the cue-words were meant to generate a past event in a flexible way. Based on Addis, Wong, and Schacter (2007), we distinguished two phases in the evocation of the event:

(1) the construction phase corresponding to the search and initial building up of the event and

(2) the elaboration phase corresponding to the retrieval of details associated with the event. Each trial had a fixed duration of $20 \mathrm{~s}$. Once the memory was retrieved, the subject had to press button 1 on the response box to mark the end of the construction phase. The remaining time, during which a central fixation cross was presented, was devoted to the elaboration phase.

The control task was a categorical task, adapted from the initial fMRI paradigm by Addis et al. (2007) for its use with patients. Thirty-two pairs of words were included, with which participants had to construct a sentence for the construction phase (e.g., with "cake-chocolate": "The pastry chef's speciality is the chocolate cake"). Once the sentence was constructed, they had to press button 1 to pursue with the elaboration phase during which the participants had to keep the same sentence structure, replacing the two given cue-words by words of the same semantic category (e.g., "The pastry chef's speciality is the apple pie"). The fixed duration for each trial was also $20 \mathrm{~s}$.

Prior to scanning, the procedure was explained to the participants and we asked them to complete a computerised practice trial for each task in order to be familiar with the experimental design and timing presentation of stimuli. Given the fact that the fMRI examination imposes time constraints, a particular attention was paid to the patients' practice trial to optimise the further completion of the task during the scanning session. Additionally, a more specific practice trial for the control task was carried out to instruct the participants to avoid any self-implication in the sentence construction (no sentence with "I"), since this process was of interest in the experimental condition.

The experimental design was organised in four sequences of eight stimuli per condition, beginning systematically with the control task and then alternating between past events and control task conditions. In both tasks, each trial was followed by a short period of fixation that was of jittered duration (mean duration $=1.5 \mathrm{~s}$, range $=1-2 \mathrm{~s}$ ). At the beginning of each sequence, the name of the condition was displayed on the screen for $6 \mathrm{~s}$. The presentation order of stimuli within each condition was randomised.

The programming and response collection were done with E-Prime 2 software (Psychology Software Tools, Inc.). Words were displayed on a screen in white text, with a black background and viewed using a mirror incorporated in the head-coil.

Immediately following scanning, a post-scan questionnaire was completed for the past events condition, in order to verify the adequacy of the responses and to exclude incorrect trials before the data analysis. Thus, for each past event, participants had to indicate the type of memory (unique, repetitive, extensive, semantic or absent), the spatial and temporal context of the event, the emotional valence and rate, on two visual-analogue scales of 10 $\mathrm{cm}$, the difficulty of access and the amount of details.

\section{Magnetic resonance imaging acquisition}

Magnetic resonance imaging (MRI) examinations were performed on a 3T MRI scanner (MAGNETOM Verio, Siemens Healthcare, Erlangen, Germany). Regarding the structural images, they were obtained by means of a 3D 
TI-weighted sampling perfection with application optimised contrasts using different flip angle evolution sequence $\left(\mathrm{TR}=4000 \mathrm{~ms}, \mathrm{TI}=380 \mathrm{~ms}, \mathrm{TE}=383 \mathrm{~ms}\right.$, flip angle $=120^{\circ}, \mathrm{FOV}=256 \mathrm{~mm}$, matrix $=512 \times 512$, 176 sagittal slices of $1 \mathrm{~mm})$. 3D T2 Fast Spin Echo images were also acquired with the following parameters: $\mathrm{TR}=3200 \mathrm{~ms}, \mathrm{TE}=409 \mathrm{~ms}$, flip angle $=120^{\circ}, \mathrm{FOV}=256 \mathrm{~mm}$, matrix $=512 \times 512,176$ sagittal slices of $1 \mathrm{~mm}$.

Functional images were acquired with a T2*-weighted echo planar imaging sequence $(\mathrm{TR}=2500 \mathrm{~ms}, \mathrm{TE}=30$ $\mathrm{ms}$, matrix $=64 \times 64$ voxels, FOV $=224 \mathrm{~mm}, \mathrm{FA}=90$ ). The anterior commissure and posterior commissure were identified in the midsagittal slice, and 45 contiguous slices (each $4 \mathrm{~mm}$ thick) were prescribed parallel to the anterior commissure and posterior commissure plane to cover the whole brain.

\section{Statistical analyses}

\section{Behavioural data}

Although AM impairment was an inclusion criteria for patients (based on normative data), between-group comparisons for the AI scores with the control group recruited for the present study were conducted ( $t$-test). This analysis was performed in order to verify that our group of MS patients actually showed lower AM scores in comparison with the sample of matched healthy controls, obtained for the present study. Between-group comparisons for the post-scan variables were also tested by means of $t$-test or repeated measures analysis of variance.

\section{Neuroimaging data}

Functional MRI image analysis. Pre-processing and statistical analyses were conducted using SPM5 software (Wellcome Department of Cognitive Neurology, London, UK, Friston et al., 1995). Time series were realigned to the first volume to correct for motion artefacts, spatially normalised to a standard EPI template based on the Montreal Neurological Institute reference brain in Talairach space (Talairach \& Tournoux, 1988), and then spatially smoothed using an $8 \mathrm{~mm}$ full-width at half-maximum isotropic Gaussian kernel.

For the past events condition and the control task, evoked hemodynamic responses time locked to the onset of the cue presentation (construction phase) were modelled with a canonical hemodynamic response function. Hemodynamic activity related to the elaboration phase was modelled with a boxcar function of 10-s duration that started immediately after the end of the construction phase by button press. The 10-s duration interval for the elaboration phase was fixed to allow a sufficient time interval to detail search and the trials with a construction phase longer than $10 \mathrm{~s}$ were excluded.

To obtain a sufficient number of valid trials for the past condition (particularly given the patients' AM impairment), we chose to keep both unique and repetitive events as regressors of interest, especially as a great overlap of the cerebral activations was previously observed for these two types of memories (Addis, McIntosh, Moscovitch, Crawley, \& McAndrews, 2004; Addis, Moscovitch, et al., 2004). Valid control trials (i.e., with a construction phase no longer than $10 \mathrm{~s}$ ) were also included as regressors of interest.

Statistical parametric maps were generated for the comparison between the past and control conditions, during the construction and elaboration phases, for each subject, with a general linear model. Whole brain betweengroup comparisons (patients > healthy controls and patients < healthy controls) were conducted by means of two-sample $t$-tests for each contrast images (past event construction vs control construction; past event elaboration vs control elaboration).

Parametric modulation analyses were performed for the difficulty of access and amount of details, with the creation of a general linear model for the construction and elaboration phases, using the first-order parametric modulation option supplied by SMP5. To this end, to obtain a homogenous distribution of the responses, ratings from the two visual analogue-scales were rescheduled empirically as follows: $0-5 \mathrm{~cm}=1$ (low score); $5.1-7.5 \mathrm{~cm}$ $=2$ (medium score) and 7.6-10 cm = 3 (high score). Two sample $t$-tests were then applied to explore the potential between-group differences.

To provide a good balance between controlling the Type I and II error rates, the significance threshold was set at $p=.005$ (uncorrected for multiple comparisons), with a minimum extent threshold of 10 contiguously activated voxels for all the analyses ( $k=10$ voxels; Lieberman \& Cunningham, 2009). Given that the medial temporal lobe structures (parahippocampal gyrus and hippocampus) were of a priori interest and that they are particularly small brain regions, explorative analyses were also conducted with a minimum extent threshold of five contiguously activated voxels ( $k=5$ voxels; $p=.005$ uncorrected), similarly to what has been done in previous AM studies (e.g., Holland, Addis, \& Kensinger, 2011).

Structural MRI image analysis. To investigate global brain tissue atrophy, segmentation of grey and white matter was performed in SPM12b (Statistical Parametric Mapping, http://www.fil. ion.ucl.ac.uk/spm/) using an extension of the unified segmentation procedure (Ashburner \& Friston, 2005) that includes six classes of tissue. 
Then, between-group differences for the grey and white matter global volumes were calculated by means of unilateral $t$-test for independent samples. For all these statistical analyses, differences were considered significant at $p<.05$. Brain lesions were manually segmented on both T1-weighed and T2-weighted images using the ITKsnap software (www.itksnap.org) for each MS patient.

\section{RESULTS}

\section{Behavioural results}

The patients' neuropsychological baseline scores were in the normal range (i.e., threshold: either $z$-score -1.65 or the 5th percentile, depending on the normative data provided for each test) for all cognitive functions, with two exceptions, planning abilities and cognitive estimations (Table 2).

With regard to the AI performance, the MS patients and the healthy control group provided respectively, on average, 14.71 internal details $(\mathrm{SD}=3.56)$ vs $29.11(\mathrm{SD}=7.56)$, with a mean total ratings of $4.12(\mathrm{SD}=1.11)$ vs $7.71(\mathrm{SD}=1.63)$ during the free recall phase. These descriptive results revealed that our group of MS patients provided a mean number of internal details approximately two SDs below those provided by healthy controls in the normative database provided by Ernst et al. (2012). In this context, as expected, the patients' performance was significantly lower than that of the present group of healthy controls for the mean number of internal details $(t=-7.31 ; p<.001)$ and the mean total ratings $(t=-7.74 ; p<.001)$.

Turning to the behavioural results of the neuroimaging session (Table 3), there was no group difference in the mean reaction time to retrieve a memory $(t=0.87 ; p=.39)$. Post-scan results highlighted a main effect of the event type $(F=123.30 ; p<.001)$, and Tukey HSD post-hoc test revealed a higher number of unique events in comparison with the four other event types $(p<.001$ in all the cases). Nevertheless, a comparable mean number of unique $(p=.58)$, repetitive $(p=1.0)$, extended $(p=.99)$, semantic $(p=.99)$ and absent $(p=088)$ memories between the two groups was found. A main effect of the emotional valence was observed $(F=63.67 ; p<.001)$, and Tukey HSD post-hoc test revealed a higher number of positive past events in comparison with neutral and negative events $(p<.001$ in all the cases). However, a similar distribution between positive $(p=.81)$, neutral $(p$ $=.48)$ and negative past events $(p=.99)$ was observed in the two groups. The mean rating for the difficulty of access $(t=.99 ; p=.33)$ and for the amount of details $(t=-.57 ; p=.57)$ did not differ between the two groups.

TABLE 2: Neuropsychological baseline examination scores: mean (and SD) for the MS patient group

\begin{tabular}{ll}
\hline & MS group score \\
\hline Verbal IQ & $99.78(11.94)$ \\
PM12 & $9.28(1.78)$ \\
RAVLT & \\
Total mean number of words & $11.96(1.35)$ \\
Delayed recall & $13.28(2.11)$ \\
ROCF & \\
Copy & $35.69(0.67)$ \\
fmmediate recall & $24.78(5.34)$ \\
Delayed recall & $24.06(5.71)$ \\
Deno 100 & $97.39(4.16)$ \\
Stroop & \\
Colours (score T) & $47.22(9.16)$ \\
Words (score T) & $42.44(12.61)$ \\
Interference (score T) & $46.50(10.10)$ \\
Interference score (score T) & $48.61(7.73)$ \\
Months back (sec) & $11.11(2.78)$ \\
Tower of London & \\
Score & $8.33(1.71)^{\mathrm{a}}$ \\
Time indice & $16.94(2.94)$ \\
Brixton (number of errors) & $14.22(4.47)$ \\
Cognitive estimation task & $4.06(2.55)^{\mathrm{a}}$ \\
Verbal fluency & \\
Categorical & $19.53(4.81)$ \\
Phonological & $13.00(3.06)$ \\
Information processing speed & \\
Cognitive & $55.11(8.31)$
\end{tabular}




$\begin{array}{ll}\text { Motor } & 49.83(10.05) \\ \text { Error percentage } & 2.61(2.68) \\ \text { Corrected score } & 60.92(9.45) \\ \text { VOSP } & \\ \text { Silhouettes } & 22.83(2.73) \\ \text { Cubes analysis } & 9.72(0.57) \\ \text { MADRS } & 5.00(4.42) \\ \text { EMIF-SEP (total) } & 44.93(15.53)\end{array}$

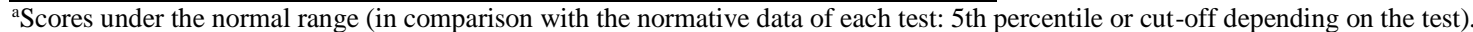
PM12, progressive matrices 12; RAVLT, Rey auditory verbal learning test; ROCF, Rey-Osterrieth complex figure; VOSP, visual object and space perception; MADRS, Montgomery and Asberg Depression Rating Scale; EMIF-SEP, Echelle de Mesure de l'Impact de la Fatigue.

TABLE 3: Mean (and standard deviation) for the behavioural measures from the fMRI session for the MS patient and the healthy control groups

\begin{tabular}{lcc}
\hline Construction reaction time (in sec) & MS patients & Healthy controls \\
Type of events & $5.40(0.93)$ & $5.07(1.28)$ \\
Unique & & \\
Repetitive & $17.39(5.48)$ & $19.83(6.00)$ \\
Extended & $4.22(4.05)$ & $4.00(3.54)$ \\
Semantic & $0.61(0.91)$ & $1.27(1.84)$ \\
Absent & $1.22(2.82)$ & $0.50(1.10)$ \\
Emotional valence & $4.61(4.38)$ & $2.77(1.83)$ \\
Positive & & \\
Neutral & $12.83(5.04)$ & $14.28(3.00)$ \\
Negative & $3.22(3.87)$ & $5.27(2.65)$ \\
Difficulty of access (parametric) & $5.94(2.75)$ & $5.50(2.77)$ \\
Amount of details (parametric) & $2.45(0.38)$ & $2.34(0.30)$ \\
\hline
\end{tabular}

\section{Structural neuroimaging results}

Regarding global brain tissue atrophy analysis, within the group of MS patients, the mean grey matter volume was $570.166 \mathrm{~cm}^{3}\left(\mathrm{SD}=64.49 \mathrm{~cm}^{3}\right)$ and the mean white matter volume was $378.12 \mathrm{~cm}^{3}\left(\mathrm{SD}: 68.70 \mathrm{~cm}^{3}\right)$. For the healthy controls, a mean grey matter volume of $613.06 \mathrm{~cm}\left(\mathrm{SD}=77.11 \mathrm{~cm}^{3}\right)$ was observed, together with a mean white matter volume of $404.86 \mathrm{~cm}^{3}\left(\mathrm{SD}=50.69 \mathrm{~cm}^{3}\right)$. These descriptive results revealed that, on average, the MS patients showed a decrease in their grey and white matter volumes of approximately $7 \%$, relatively to the healthy subjects. Statistical analyses confirmed the presence of a significant decrease in the grey matter volume in MS patients, relatively to the healthy controls $(t=-1.75, p=.04)$. However, for the white matter volume, this difference did not reach the statistical significance threshold $(t=-1.30, p=.10)$.

In parallel, regarding the total lesion load, within the patient group, the mean lesion volume was $4.50 \mathrm{~cm}^{3}(\mathrm{SD}=$ $\left.5.10 \mathrm{~cm}^{3}\right)$.

\section{Functional neuroimaging results}

\section{Past events construction}

During the initial construction phase, between-group analysis revealed significantly greater activations in MS patients than in healthy controls in several brain areas (Table 4; Figure 1). These increased cerebral activations were predominantly located in the frontal regions, including the right inferior frontal gyrus (BA 46), the right middle frontal gyrus (BA 10), the right superior frontal gyrus (BA 6) and the left precentral gyrus (BA 6/ 43/44), and also in the left transverse temporal gyrus (BA 42), the left postcentral gyrus (BA 40), the right postcentral gyrus (BA 2) and the left insula (BA 13).

The reverse contrast (healthy controls vs MS patients) showed significantly greater brain activations only in the right superior temporal gyrus (BA $13 ; x=46, y=0, z=-8, Z=2.79$ ).

\section{Past events elaboration}

Differential activations between MS patients and healthy controls were also observed during the elaboration on additional details from past events (Table 4; Figure 2). MS patients recruited more extensively the frontal 
regions, with significant activations in the right middle frontal gyrus (BA 8/9/10), the left middle frontal gyrus (BA 8/ 46), the right inferior frontal gyrus (BA 47), the right superior frontal gyrus (BA 9), the left superior frontal gyrus (BA 9/10) and the bilateral medial frontal gyrus (BA 9). Increased activations were also observed in the left middle (BA 21) and superior (BA 42) temporal gyri, the right middle occipital gyrus (BA 18), the left cingulate gyrus (BA 25/32), the right cingulate gyrus (BA 23/24/32), the right cuneus (BA 17), the left precuneus (BA 7) and the right cerebellum and in basal nuclei (bilateral thalamus, bilateral caudate nuclei, left putamen).

The healthy controls did not show significantly more brain activation than the patients in any brain regions.

Parametric modulation of brain activity during past events construction

Differential neural responses to the difficulty of access and to the amount of details were highlighted between MS patients and healthy controls during retrieval of past events (Tables 5 and 6; Figure 3A.1 and A.2).

Indeed, for the patients, the response to the difficulty of access entailed a greater activity only in the right parahippocampal gyrus (BA 19) in comparison with the healthy controls. The reverse contrast showed increased activations for the healthy controls in the bilateral postcentral gyrus (BA 7), the right superior parietal lobule (BA 7), the left middle frontal gyrus (BA 6), the right middle temporal gyms (BA 21), the left lingual gyms (BA 19) and the left cerebellum, in response to the difficulty of access.

Concerning the amount of details, between-group analysis showed a significantly greater modulation effect for the patients in the left medial frontal gyrus and the right uncus (BA 28), whereas the healthy controls recruited more extensively the left superior temporal gyrus (BA 13) in response to the quantity of details.

TABLE 4: Brain regions exhibiting significant activations for MS patients (vs healthy controls; two sample Mest) during the past event construction (vs control construction) and the past event elaboration (vs control elaboration)

\begin{tabular}{lcc}
\hline Brain region & $\begin{array}{c}\text { Coordinates } \\
(x, \mathrm{y}, \mathrm{z})\end{array}$ & $\mathrm{Z}-$ score \\
\hline Past event construction > control construction & \\
L Precentral gyrus (BA 43) & $(-54,-8,12)$ & 2.85 \\
L Precentral gyrus (BA 44) & $(-60,10,8)$ & 2.95 \\
L Precentral gyrus (BA 6) & $(-58,0,6)$ & 2.74 \\
R Inferior frontal gyrus (BA 46) & $(52,42,2)$ & 3.15 \\
R Middle frontal gyrus (BA 10) & $(34,56,6)$ & 2.98 \\
R Superior frontal gyrus (BA 6) & $(24,6,70)$ & 2.96 \\
L Transverse temporal gyrus (BA 42) & $(-60,-18,14)$ & 3.58 \\
L Postcentral gyrus (BA 40) & $(-56,-26,20)$ & 3.48 \\
R Postcentral gyrus (BA 2) & $(48,-36,64)$ & 2.95 \\
L Insula (BA 13) & $(-42,14,24)$ & 2.78 \\
Past event elaboration > control elaboration & & \\
R Middle frontal gyrus (BA 10) & $(30,40,22)$ & 3.30 \\
L Superior frontal gyrus (BA 10) & $(-2,66,24)$ & 3.30 \\
R Inferior frontal gyrus (BA 47) & $(16,10,-14)$ & 2.92 \\
R Middle frontal gyrus (BA 8) & $(22,24,38)$ & 2.85 \\
L Middle frontal gyrus (BA 8) & $(-32,34,48)$ & 3.0 \\
R Middle frontal gyrus (BA 9) & $(40,46,26)$ & 3.58 \\
R Superior frontal gyrus (BA 9) & $(8,54,28)$ & 3.56 \\
L Medial frontal gyrus (BA 9) & $(-6,44,26)$ & 3.07 \\
L Middle frontal gyrus (BA 46) & $(-42,40,14)$ & 3.24 \\
L Superior frontal gyrus (BA 9) & $(-24,38,30)$ & 3.05 \\
R Medial frontal gyrus (BA 9) & $(12,26,30)$ & 2.82 \\
R Middle occipital gyrus (BA 18) & $(22,-92,4)$ & 3.62 \\
L Middle temporal gyrus (BA 21) & $(-34,4,-30)$ & 3.67 \\
L Superior temporal gyrus (BA 42) & $(-60,-20,12)$ & 2.70 \\
L Thalamus & $(-18,-12,16)$ & 3.21
\end{tabular}


$\mathrm{R}$ Thalamus

L Anterior cingulate (BA 25)

$\mathrm{R}$ Anterior cingulate (BA 32)

R Cingulate gyrus (BA 24)

L Cingulate gyrus (BA 32)

R Posterior cingulate (BA 23)

R Cuneus (BA 17)

L Precuneus (BA 7)

$\mathrm{R}$ Cerebellum anterior lobe

$\mathrm{R}$ Cerebellum posterior lobe

R Caudate nucleus

L Caudate nucleus

L Putamen

$\begin{array}{cc}(2,-4,2) & 3.08 \\ (-6,14,-8) & 2.72 \\ (10,20,-8) & 3.47 \\ (10,-8,32) & 2.79 \\ (0,24,34) & 2.75 \\ (6,-38,26) & 3.21 \\ (16,-84,6) & 2.67 \\ (-8,-50,60) & 3.00 \\ (24,-52,-24) & 3.51 \\ (24,-60,-46) & 3.45 \\ (18,12,8) & 2.89 \\ (-20,2,22) & 3.67 \\ (-20,10,14) & 3.23\end{array}$

Talairach coordinates reported. Uncorrected $p<.005 ; k=10$ voxels.

Figure 1. Brain regions showing an increased activity for MS patients (vs healthy controls) during the past events construction (vs control task construction); uncorrected $p<.005, k=10$ voxels.

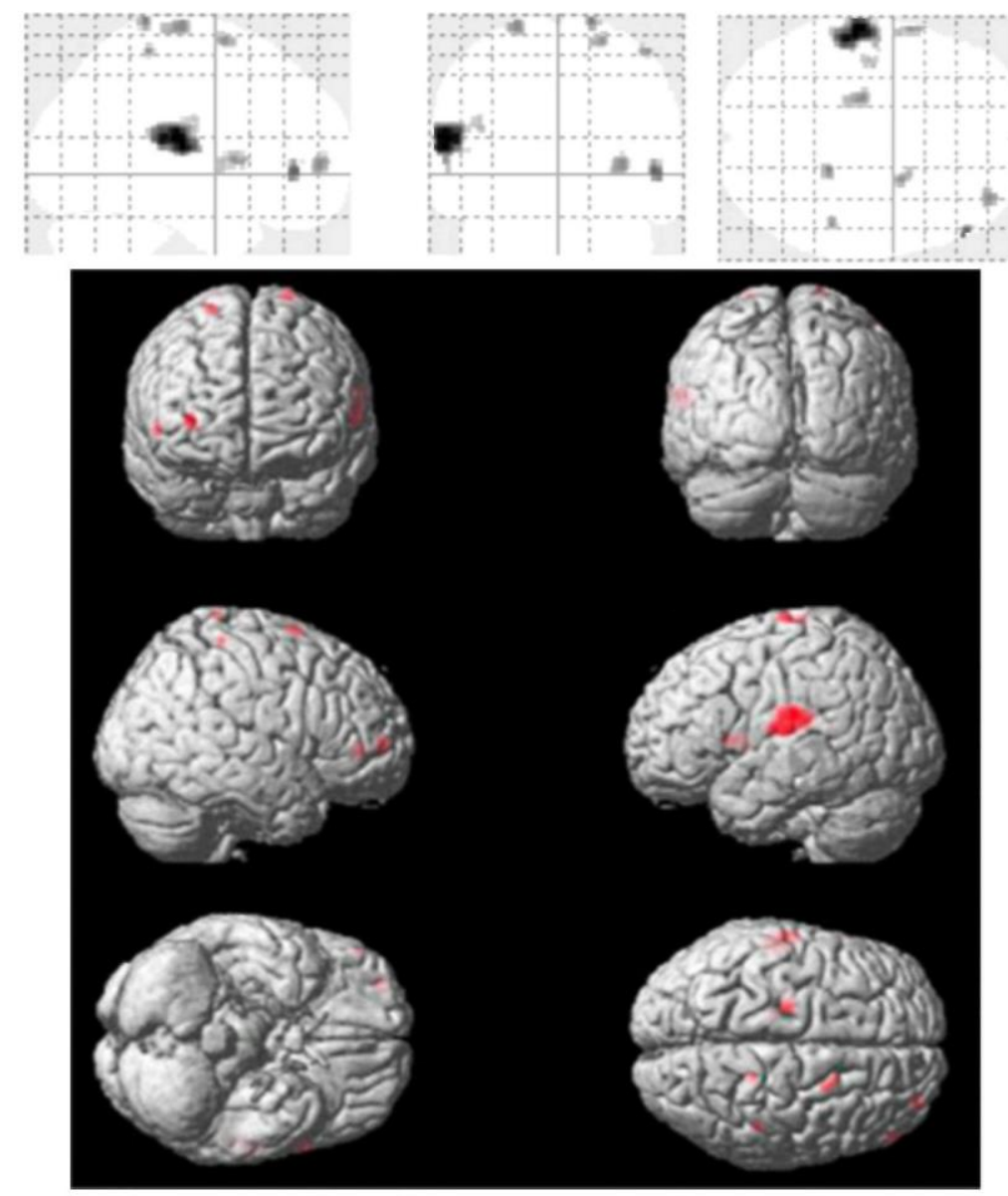

Parametric modulation of brain activity during past events elaboration

During the elaboration phase, the parametric response for the difficulty of access and amount of details involved different brain regions in the two groups (Tables 5 and 6; Figure 3B.1 and B.2).

MS patients showed a greater response to the difficulty of access in the left parahippocampal gyrus (BA 30), the right parahippocampal gyrus (BA 27) and in the left middle temporal gyrus (BA 22) in comparison with the healthy controls. Analysis with $k=5$ highlighted also a significant activation in the right hippocampus. 
Conversely, relatively to the patients, controls' neural responses to the difficulty of access led to greater leftlateralised activations in the lingual gyrus (BA 19), the middle temporal gyrus (BA 19) and the cuneus (BA 17).

Regarding the neural responses to the amount of details, the patients recruited more intensively than the controls right-sided brain regions, with significant activations in the parahippocampal gyrus (BA 37), the fusiform gyrus (BA 37) and the cingulate gyrus (BA 31). Again, analysis with $k=5$ revealed a significant activation in the right hippocampus. The reverse contrast showed a significantly greater activation in the right middle temporal gyrus (BA 39) within the group of healthy controls, in response to the amount of details.

Figure 2. Brain regions showing an increased activity for MS patients (vs healthy controls) during the past events elaboration (vs control task elaboration); uncorrected $p<.005, k=10$ voxels.

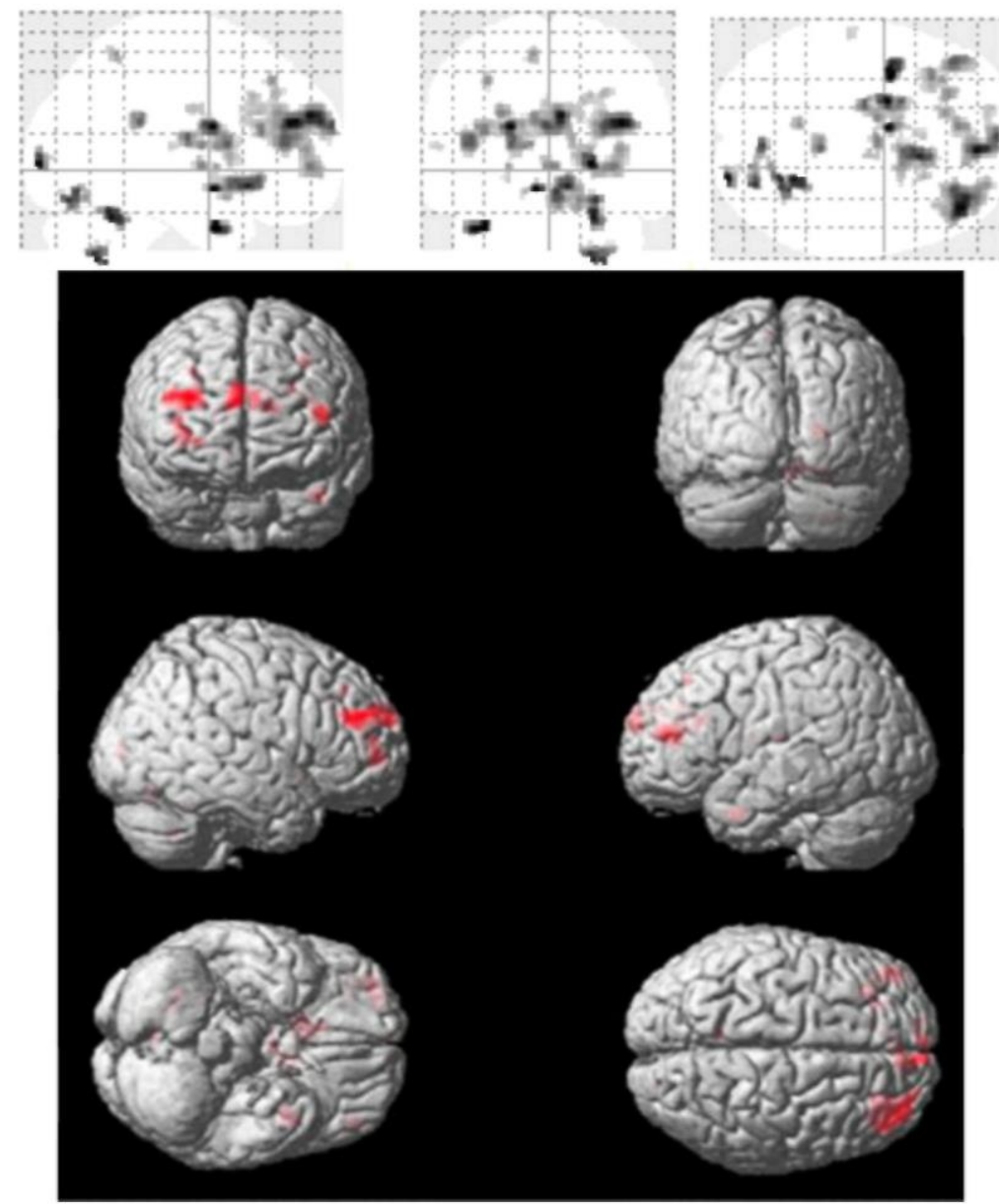

\section{DISCUSSION}

In the present study, we have shown that AM impairment observed at the clinical level in MS patients was accompanied by cerebral activation changes during the AM fMRI task, in the context of a significant grey matter loss.

Importantly, notwithstanding the above-mentioned AM clinical impairment, our MS patients were still able to perform the AM fMRI task at a comparable level to that of the healthy control group (Maguire et al., 2001). Therefore, the observed cerebral activation changes in the patient group are not to be interpreted as reflecting, for instance, a too poor completion of the task in comparison with the healthy controls (Cader, Cifelli, Abu-Omar, Palace, \& Matthews, 2006; Liu et al., 2011; Maguire et al., 2001). While this result could seem counterintuitive, especially for the type of events, difficulty of access and amount of details, there are a number of factors at work. Indeed, since one criterion for patients' inclusion was impaired score on the AI, we paid particular attention to the practice trial preceding the fMRI session to optimise the procedural aspects accompanying the task itself. Moreover, considering the issue of the AM test sensitivity, it is possible that the simple categorisation of the type 
of events and the two visual-analogue scales were not sensitive enough to detect deficits similar to that observed in the patients' AI In relation, again, to the issue of the level of difficulty, qualitatively, nearly all the patients reported that the AM fMRI task was easier than the AI Furthermore, it should be noted that the post-scan measures relied on self-assessment, which could imply the influence of different decision criteria within the participants, whereas the AI is an objective measure of the number of episodic details provided by the subjects. Importantly, in coherence with Ernst et al.'s data $(2012,2013,2014)$, showing that MS patients expressed AM difficulties congruent with objective AM assessment, self-assessment reliability in the present study was based on the fact that the patients demonstrated AM impairment in the context of preserved general cognitive functioning, and normal awareness of their AM difficulties (qualitatively observed during both the AI and the post-scan questionnaire)

While cerebral activation changes have already been reported in different cognitive domains in MS patients (e.g., Hulst et al., 2012; Kollndorfer et al., 2013; Morgen et al., 2007), the present study is the first, to our knowledge, to provide evidence of functional activation changes during recollection of AMs. Specifically, these brain activation changes in MS patients were mainly located in key brain regions of the AM core network, which is in agreement with the previous findings obtained in different clinical conditions presenting with AM deficit (Maguire et al., 2001, 2010; Meulenbroek et al., 2010).

Interestingly, these brain activation changes in our patients were expressed by both increased cerebral activations in key brain regions of the AM core brain network and the recruitment of alternative, mostly contralateral, brain areas. Overall, in our MS patients, predominant brain activation changes were demonstrated in the bilateral prefrontal regions, in accord with previous preliminary findings (Ernst et al., 2012). This result could be accounted for the significant role of the frontal network in AM retrieval (Svoboda et al., 2006) and it is coherent with the retrieval deficit hypothesis posited by Ernst et al. $(2012,2013,2014)$ to explain AM impairment in MS patients.

More precisely, regarding the construction phase of AM, the patients exhibited predominantly functional changes in the bilateral frontal regions. The right-lateralised activations are particularly significant since the AM core network is predominantly left-lateralised (Addis et al., 2007; Botzung, Denkova, Ciuciu, Scheiber, \& Manning, 2008). These frontal region activations were also accompanied by increased bilateral parietal activations. The greater activity of this bilateral frontoparietal network is of interest considering that, besides the well-known role of frontal regions in AM retrieval (Cabeza \& St Jacques, 2007; Svoboda et al., 2006), an involvement of parietal regions in the early phase of AM has also been described (Addis et al., 2007; Berryhill, Phuong, Picasso, Cabeza, \& Olso, 2007). Along these lines, St Jacques, Kragel, and Rubin, 2011 introduced the notion of the left "frontoparietal network" in AM. Given that this last network is related to both executive control processes (Niendam et al., 2012; Vincent, Kahn, Snyder, Raichle, \& Buckner, 2008;) and the Attention to Memory model (Cabeza, 2008), it has been suggested that its role is linked to the important demand of top-down attentional control processes during the construction of memories. This frontoparietal network could be especially involved in memory retrieval "when judgements are uncertain and that further analysis of the stimulus in relation to mnemonic representations is required" (Vincent et al., 2008; p. 3338). On these bases, we suggest with caution that, taking into account the relationships between AM deficit and executive functioning in MS patients (Ernst et al., 2013), the enhanced recruitment of the bilateral frontoparietal regions could reflect the higher cognitive demand during the construction phase in MS patients.

Regarding the posterior brain regions, Addis et al. (2007) reported early cerebral activations in these brain areas during the construction phase, which is consistent with the role of visual imagery in the retrieval and vividness of episodic memories (Greenberg \& Rubin, 2003). The increased recruitment of these regions, only observed during the further elaboration phase in our patients, together with the aforementioned primary focus on executive demand during the construction phase, could suggest that the visual imagery processes might have been "delayed" in our patients. In fact, during the elaboration phase, our group of patients showed a remarkably enhanced activity in the right middle occipital gyrus, the right cuneus and the left precuneus.

Similar to the construction phase, our patients exhibited important cerebral activations in the bilateral prefrontal regions during the elaboration phase, located in the middle and superior frontal gyri and the medial frontal regions. In relation to the elaboration phase, Addis et al. (2007) underlined the recruitment of predominantly left prefrontal regions, which are involved in the further retrieval, verification and selection of information required during the elaboration of memories (Svoboda et al., 2006). In parallel, the left medial prefrontal region is particularly engaged in self-referential process (Cabeza \& St Jacques, 2007; Gilboa, 2004; Maguire, 2001).

Turning to the cortical midline brain structures, Summerfield, Hassabis, and Maguire (2009) have proposed that the right posterior cingulate cortex activity is modulated by the realness and self-implication during memory recollection. In addition, Northoff et al. (2006) have suggested that the anterior cingulate cortex is associated with the recall of self-events and may mediate the interaction between the emotional significance and the self- 
relevance of stimuli (Moran, Macrae, Heatherton, Wyland, \& Kelley, 2006; Sharot, Riccardi, Raio, \& Phelps, 2007). Not surprisingly, these brain regions are particularly active during the elaboration phase of memories (Addis et al., 2007). In the present study, we observed a greater recruitment of the bilateral anterior cingulate cortex and the right posterior cingulate cortex, relatively to the healthy controls.

Regarding the temporal regions, we observed an enhanced activity in the left middle temporal gyrus (BA 21) associated with AM semantic knowledge (Svoboda et al., 2006) but no contral-ateral recruitment. Based on the relative preservation of personal semantics in MS (Ernst et al., 2013), we suggest with caution that personal semantic representations could play a role in compensating AM recollection difficulties, manifested by the enhanced activity in this region. Based on previous reports showing hippocampal damage in MS patients (Hulst et al., 2012; Sicotte et al., 2008) and the central role of the hippocampus in AM (Addis, Moscovitch et al., 2004; Addis, Mclntosh et al., 2004; Addis \& Schacter, 2008), functional changes in the hippocampal region were hypothesised in the present study. Surprisingly, no significant between-group differences were found regarding the hippocampal activations.

TABLE 5: Significant brain activations for the parametric modulation of the difficulty of access and amount of details for MS patients (vs healthy controls; two sample t-est) during the past event construction and elaboration

\begin{tabular}{lcc} 
Brain region & $\begin{array}{c}\text { Coordinates } \\
(x, \mathrm{y}, \mathrm{z})\end{array}$ & $z$-score \\
\hline Past event construction — difficulty of access & & \\
$\begin{array}{l}\text { R Parahippocampal gyms (BA 19) } \\
\text { Past event construction - amount of details }\end{array}$ & $(34,-48,-2)$ & 2.93 \\
L Medial frontal gyms & $(-16,58,2)$ & 3.22 \\
R Uncus (BA 28) & $(28,-10,-26)$ & 2.87 \\
Past event elaboration - difficulty of access & & \\
L Middle temporal gyms (BA 22) & $(-36,-54,18)$ & 3.07 \\
L Parahippocampal gyms (BA 30) & $(-30,-48,8)$ & 2.97 \\
R Parahippocampal gyms (BA 27) & $(22,-34,0)$ & 2.86 \\
Past event elaboration—amount of details & & \\
R Parahippocampal gyms (BA 37) & $(32,-38,-10)$ & 3.29 \\
R Fusiform gyms (BA 37) & $(44,-42,-12)$ & 2.89 \\
R Cingulate gyms (BA 31) & $(24,-28,40)$ & 3.00 \\
\hline
\end{tabular}

Talairach coordinates reported. Uncorrected $p<.005 ; k=10$ voxels.

TABLE 6: Significant brain activations for the parametric modulation of the difficulty of access and amount of details for healthy controls (vs MS patients; two sample Mest) during the past event construction and elaboration

\begin{tabular}{|c|c|c|}
\hline Brain region & $\begin{array}{c}\text { Coordinates } \\
(x, \mathrm{y}, \mathrm{z})\end{array}$ & $\mathrm{z}$-score \\
\hline \multicolumn{3}{|c|}{ Past event construction - difficulty of access } \\
\hline L Postcentral gyms (BA 7) & $(-22,-48,72)$ & 3.55 \\
\hline R Superior parietal lobule (BA 7) & $(36,-72,52)$ & 3.19 \\
\hline R Postcentral gyrus (BA 7) & $(6,-52,72)$ & 3.12 \\
\hline L Middle frontal gyrus (BA 6) & $(-36,2,64)$ & 3.29 \\
\hline R Middle temporal gyrus (BA 21) & $(56,0,-18)$ & 3.09 \\
\hline L Lingual gyrus (BA 19) & $(-18,-66,0)$ & 3.09 \\
\hline L Cerebellum posterior lobe & $(-20,-74,-16)$ & 3.00 \\
\hline \multicolumn{3}{|c|}{ Past event construction - amount of details } \\
\hline L Superior temporal gyrus (BA 13) & $(-52,-44,16)$ & 3.49 \\
\hline \multicolumn{3}{|c|}{ Past event elaboration-difficulty of access } \\
\hline L Lingual gyrus (BA 19) & $(-20,-66,-2)$ & 3.34 \\
\hline L Middle temporal gyrus (BA 19) & $(-38,-82,20)$ & 3.09 \\
\hline L Cuneus (BA 17) & $(-12,-80,6)$ & 2.83 \\
\hline \multicolumn{3}{|c|}{ Past event elaboration-amount of details } \\
\hline R Middle temporal gyrus (BA 39) & $(46,-72,26)$ & 2.97 \\
\hline
\end{tabular}

Talairach coordinates reported. Uncorrected $p<.005 ; k=10$ voxels. 
Figure 3. Brain regions showing an increased response to parametric modulation for MS (vs healthy controls) during the construction $(A)$ and the elaboration $(B)$ phases for the difficulty of access $(A .1$ and B.1) and the quantity of details (A.2 and B.2); uncorrected $p<.005, k=10$ voxels.

(A.1)
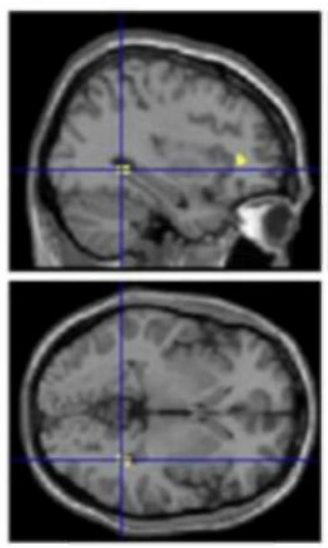

(B.1)
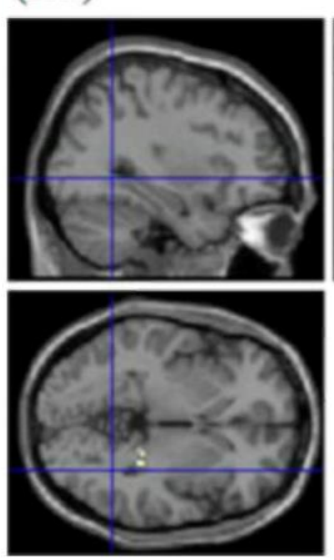

(A.2)
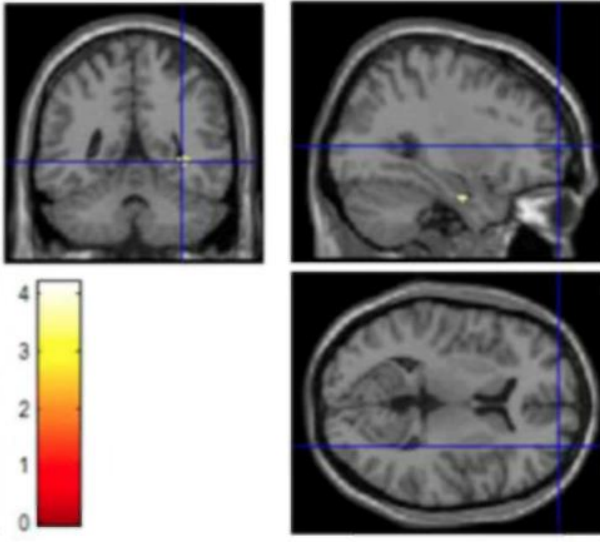

(B.2)
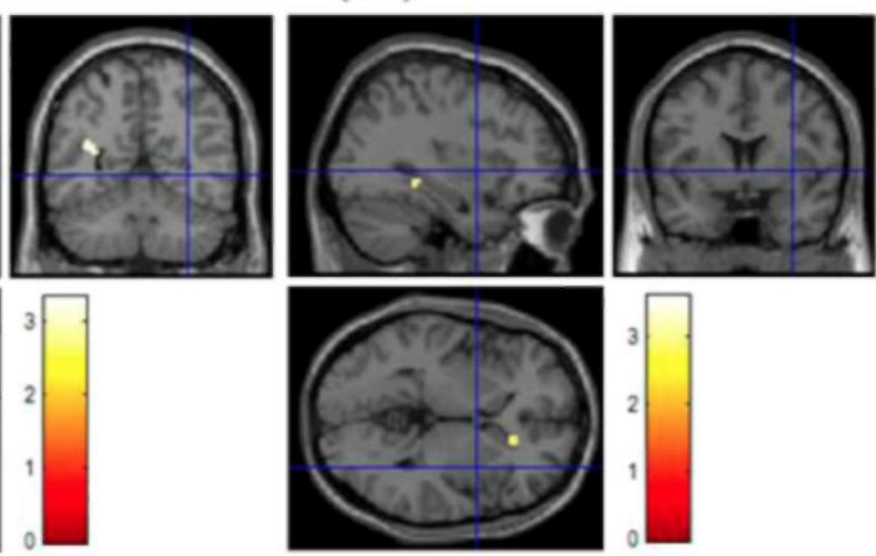
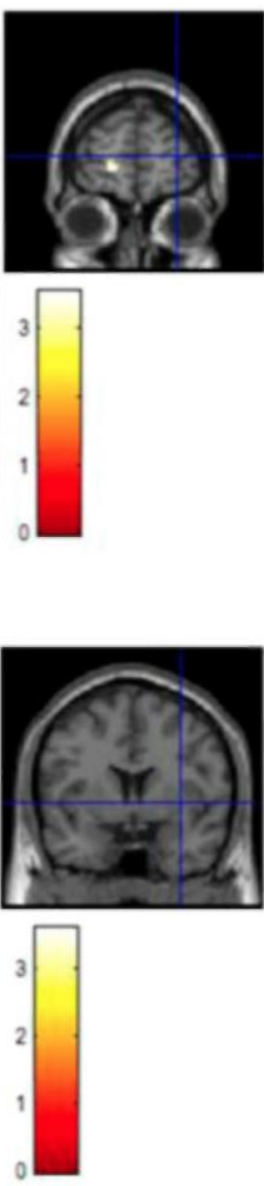

However, the introduction of parametric modulation analyses revealed a differential neural response to the recollective qualities of memories in the patient group compared with the control group, which involved some key AM regions, and especially the hippocampal region. Indeed, the difficulty of access was associated with enhanced cerebral activations in the right parahippocampal gyrus during memory construction and in the right hippocampus and the bilateral parahippocampal gyrus during elaboration. In other words, the neural activity in these brain regions increased as the difficulty of access augmented. In addition, brain activations in the right hippocam-pus/parahippocampal gyrus and in the right uncus were associated with the amount of details during the construction and elaboration phases, respectively, in our MS patients. The latter result is consistent with previous reports showing that the increase in the number of details modulates the engagement of the left hippocampus in healthy subjects (Addis, Mclntosh et al. 2004; Addis \& Schacter, 2008).

The neural activity in the middle temporal gyrus (BA 22) was also significantly modulated by the difficulty of access during the elaboration phase in the patient group. As mentioned above, this brain region is implicated in AM semantic knowledge (Svoboda et al., 2006). This finding, together with the aforementioned increased neural activity observed in this brain region during the elaboration phase, suggests that the middle temporal gyrus and personal semantics could play a particular role during the evocation of personal memories in MS patients presenting with AM impairment.

A further finding related to the number of details was the implication of the left medial frontal region and of the right posterior cingulate gyrus. As mentioned above, these brain structures support self-implication and realness of memories (Buckner, Andrews-Hanna, Schacter, 2008; Summerfield et al., 2009). Given the centrality of these phenomenological properties for episodic re-experiencing, the enhanced brain activations observed in MS patients as the amount of details increased could be of particular interest. Further investigations could address more specifically the influence of these components on AM re-experiencing in MS patients. 
With regard to the control subjects, the neural response to the difficulty of access and to the amount of details seemed to involve mostly the core regions of the AM network during the event construction, including anterior, temporal and posterior cerebral regions. These increased brain activations (in comparison with the patient group) were predominantly observed in the left hemisphere. The same applies to the elaboration phase, during which predominantly left posterior brain regions from the AM core network seemed particularly responsive.

\section{CONCLUSIONS}

In conclusion, the present study documents for the first time, to our knowledge, the presence of functional activation changes during the evocation of personal memories in patients suffering from a diffuse neurological pathology and presenting with impaired AM performance. Interestingly, these cerebral activation changes were mostly observed in key brain regions involved in AM.

Clinically, it is important to note that patients' AM complains are frequent and consistent with AM impaired performance, as previously underlined by Ernst et al. (2013, 2014). In this context, the present findings encourage the use of neuro-imaging studies to better understand AM impairment, with the ultimate goal to support the development of cognitive rehabilitation programmes. Related to the issue of rehabilitation, preliminary results with four MS patients were obtained by Ernst et al. (2012), showing significant cerebral activation changes in the context of AM improvement following a cognitive intervention. While these findings remain very preliminary, they support the use of this kind of approach to improve our understanding of cognitive impairment in patients and also the development of specific cognitive interventions in MS patients. Moreover, they encourage its application in other clinical conditions, bearing in mind that in the case of AM, the interest of these issues is of particular importance considering the deleterious impact of AM deficit in patients' everyday life. Among the reasons of such an impact is the fact that AM is ubiquitous and plays major roles in daily life functioning (Rasmussen \& Habermas, 2011).

\section{REFERENCES}

Addis, D. R., McIntosh, A. R., Moscovitch, M., Crawley, A. P., \& McAndrews, M. P. (2004). Characterizing spatial and temporal features of autobiographical memory retrieval networks: A partial least squares approach. NeuroImage, 23, 1460-1471. doi:10.1016/j.neuroimage.2004.08.007

Addis, D. R., Moscovitch, M., Crawley, A. P., \& McAndrews, M. P. (2004). Recollective qualities modulate hippocampal activation during autobiographical memory retrieval. Hippocampus, 14, 752-762. doi:10.1002/hipo.10215

Addis, D. R, Sacchetti, D. C, Ally, B. A., Budson, A. E., \& Schacter, D. L. (2009). Episodic simulation of future events is impaired in mild Alzheimer's disease. Neuropsychologia, 47, 2660-2671. doi:10.10 16/j.neuropsychologia.2009.05.018

Addis, D. R, \& Schacter, D. L. (2008). Constructive episodic simulation: Temporal distance and detail of past and future events modulate hippocampal engagement. Hippocampus, 18, 227-237. doi:10.100 2/hipo.20405

Addis, D. R, Wong, A. T., \& Schacter, D. L. (2007). Remembering the past and imagining the future: Common and distinct neural substrates during event construction and elaboration. Neuropsychologia, 45, 1363-1377. doi:10.1016/j.neuropsychologia.2006.10.016

Addis, D. R, Wong, A. T., \& Schacter, D. L. (2008). Age-related changes in the episodic simulation of future events. Psychological Science, 19(1), 33-41. doi:10.1111/j.1467-9280.2008.02043.x

Ashburner, J., \& Friston, K. J. (2005). Unified segmentation. Neurolmage, 26, 839-851. doi:10.1016/j. neuroimage.2005.02.018

Axelrod, B. N., Ryan, J. J., \& Ward, L. C. (2001). Evaluation of seven-subtest short forms of the Wechsler Adult Intelligence Scale-III in a referred sample. Archives of Clinical Neuropsychology, 16(1), 1-8. doi:10.1016/S0887-6177(99)00054-2

Barnabe, A., Whitehead, V., Pilon, R., Arsenault-Lapierre, G., \& Chertkow, H. (2012). Autobiographical memory in mild cognitive impairment and Alzheimer's disease: A comparison between the Levine and Kopelman interview methodologies. Hippocampus, 22,1809-1825. doi:10.1002/hipo.22015

Bendfeldt, K., Kuster, P., Traud, S., Egger, H., Winkl-hofer, S., Mueller-Lenke, N., ... Borgwardta, S. J. (2009). Association of regional gray matter volume loss and progression of white matter lesions in multiple sclerosis - A longitudinal voxel-based mor-phometry study. Neurolmage, 45(1), 60-67. 


\section{doi:10.1016/j.neuroimage.2008.10.006}

Benedict, R. H. B., Bakshi, R, Simon, J. H., Priore, R, Miller, C, \& Munschauer, F. (2002). Frontal cortex atrophy predicts cognitive impairment in multiple sclerosis. Journal of Neuropsychiatry and Clinical Neurosciences, 14(1), 44-51. doi:10.1176/appi.neuro psych.14.1.44

Berryhill, M. E., Phuong, L., Picasso, L., Cabeza, R, \& Olson, I. R. (2007). Parietal lobe and episodic memory: Bilateral damage causes impaired free recall of autobiographical memory. The Journal of Neuroscience, 26, 14414-14423.

Bookheimer, S. Y., Strojwas, M. H., Cohen, M. S., Saunders, A. M., Periack-Vance, M. A., Mazziotta, J. C, \& Small, G. W. (2000). Patterns of brain activation in people at risk for Alzheimer's disease. The New England Journal of Medicine, 343, 450-456. doi:10.1056/NEJM200008173430701

Botzung, A., Denkova, E., Ciuciu, P., Scheiber, C, \& Manning, L. (2008). The neural bases of the constructive nature of autobiographical memories studied with a self-paced fMRI design. Memory, 16, 351-363. doi:10.1080/09658210801931222

Buckner, R. L., Andrews-Hanna, J. R., Schacter, D. L. (2008). Episodic simulation of future events: Concepts, data, and applications. Annals of the New York Academy of Sciences, 1124, 1-38. doi: 10.1196/ annals.1440.001

Burgess, P., \& Shallice, T. (1997). The Hayling and Brixton tests. Test manual. Bury St Edmunds.: Thames Valley Test Company.

Cabeza, R. (2008). Role of parietal regions in episodic memory retrieval: The dual attention process hypothesis. Neuropsychologia, 46, 1813-1827. doi:10.10 16/j.neuropsychologia.2008.03.019

Cabeza, R., \& St Jacques, P. (2007). Functional neuroimaging of autobiographical memory. Trends in Cognitive Sciences, 11, 219-227. doi:10.1016/j. tics.2007.02.005

Cader, S., Cifelli, A., Abu-Omar, Y., Palace, J., \& Matthews, P. M. (2006). Reduced brain functional reserve and altered functional connectivity in patients with multiple sclerosis. Brain, 129, 527-537. doi:10.

1093/brain/awh670

Chen, H., Epstein, J., \& Stern, E. (2010). Neural plasticity after acquired brain injury: Evidence from functional neuroimaging. $P M \& R, 2, \mathrm{~S} 306-\mathrm{S} 312$.

Coughlan, A., \& Hollows, S. (1985). The adult memory and information processing battery. Leeds: Saint James Hospital.

D'Argembeau, A, Raffard, S., \& Van Der Linden, M. (2008). Remembering the past and imagining the future in schizophrenia. Journal of Abnormal Psychology, 117, 247-251. doi:10.1037/0021-843X.117.1.247

Debouverie, M., Pittion-Vouyovitch, S., Louis, S., \& Guillemin, F. (2007). Validity of a French version of the fatigue impact scale in multiple sclerosis. Multiple Sclerosis, 13, 1026-1032.

Ernst, A., Blanc, F., de Seze, J., Sellal, F., Chavin, B., \& Manning, L. (2014). Impaired mental simulation of specific past and future personal events in non-depressed multiple sclerosis patients. Journal of the Neurological Sciences. Advance online publication, doi: 10.1016/j.jns.2014.07.007

Ernst, A., Blanc, F., Voltzenlogel, V, De Seze, J., Chauvin, B., \& Manning, L. (2013). Autobiographical memory in multiple sclerosis patients: Assessment and cognitive facilitation. Neuropsychological Rehabilitation, 23(2), 161-181. doi:10.1080/09602011. 2012.724355

Ernst, A., Botzung, A., Gounot, D., Sellal, F., Blanc, F., de Seze, J., Manning, L. (2012). Induced brain plasticity after a facilitation programme for autobiographical memory in multiple sclerosis: A preliminary study. Multiple Sclerosis International, 2012,12. Article ID 820240. doi: 10.1155/2012/820240

Filippi, M., \& Rocca, M. A. (2004). Cortical reorganisation in patients with MS. Journal of Neurology, Neurosurgery, and Psychiatry, 75, 1087-1089. doi:10. 1136/jnnp.2004.036020

Friston, A. P., Holmes, K. J., Worsley, J. B., Poline, J. P., Frith, C. D., \& Frackowiak, R. S. J. (1995). Statistical parametric maps in functional imaging: A general linear approach. Human Brain Mapping, 2, 189-210. doi:10.1002/hbm.460020402

Gilboa, A. (2004). Autobiographical and episodic memory - one and the same? Evidence from prefrontal activation in neuroimaging studies. Neuropsychologia, 42, 1336-1349. doi:10.1016/j.neuropsychologia.2004.02.014

Greenberg, D. L., \& Rubin, D. C. (2003). The neuropsychology of autobiographical memory. Cortex, 39, 687- 


\section{8. doi:10.1016/S0010-9452(08)70860-8}

Hassabis, D., Kumaran, D., Vann, S. D., \& Maguire, E. A. (2007). Patients with hippocampal amnesia cannot imagine new experiences. PNAS, 104, 1726-1731. doi:10.1073/pnas.0610561104

Holland, A. C, Addis, D. R, \& Kensinger, E. A. (2011). The neural correlates of specific versus general autobiographical memory construction and elaboration. Neuropsychologia, 49, 3164-3177. doi:10.1016/j.neuropsychologia.2011.07.015

Hulst, H. E., Schoonheim, M. M., Roosendaal, S. D., Popescu, V, Schweren, L. J. S., van der Werf, Y. D., ... Geurts, J. J. G. (2012). Functional adaptive changes within the hippocampal memory system of patients with multiple sclerosis. Human Brain Mapping, 33, 2268-2280. doi:10.1002/hbm.21359

Irish, M., Hodges, J. R, \& Piguet, O. (2013). Episodic future thinking is impaired in the behavioural variant of frontotemporal dementia. Cortex, 49, 2377-2388. doi:10.1016/j.cortex.2013.03.002

Johansson, B. B. (2000). Brain plasticity and stroke rehabilitation: The Willis lecture. Stroke, 31, 223-230. doi:10.1161/01.STR31.1.223

Kollndorfer, K., Krajnik, J., Woitek, R., Freiherr, J., Prayer, D., \& Schöpf, V. (2013). Altered likelihood of brain activation in attention and working memory networks in patients with multiple sclerosis: An ALE meta-analysis Neuroscience and Biobehavioral Reviews, 37, 2699-2708. doi:10.1016/j.neubiorev.20 13.09.005

Kremin, H. (2002). L'accès au lexique en dénomination d'images: Problèmes actuels [Access to lexicon in linedrawing naming: Current problems]. Psychologie Française, 47, 77-91.

Kurtzke, J. F. (1983). Rating neurological impairment in multiple sclerosis: An Expanded Disability Status Scale (EDSS). Neurology, 33, 1444-1452. doi:10.121 2/WNL33.11.1444

Lazeron, R. H. C, Rombouts, S. A. R. B., Scheltens, P., Polman, C. H, \& Barkhof, F. (2004). An fMRI study of planning-related brain activity in patients with moderately advanced multiple sclerosis. Multiple Sclerosis Journal, 10, 549-555. doi:10.1191/ 1352458504ms1072oa

Levin, H. S. (2003). Neuroplasticity following non-penetrating traumatic brain injury. Brain Injury, 17, 665-674. doi:10.1080/0269905031000107151

Levine, B., Svoboda, E., Hay, J. F., Winocur, G., \& Moscovitch, M. (2002). Aging and autobiographical memory: Dissociating episodic from semantic retrieval. Psychology and Aging, 4, 677-689. doi:10.10 37/08827974.17.4.677

Leyhe, T., Muller, S., Milian, M., Eschweiler, G. W., Saur, R. (2009). Word order processing in the bilingual brain. Neuropsychologia, 47, 2464-2469. doi:10.1016/j.neuropsychologia.2008.08.007

Lieberman, M. D., \& Cunningham, W. A. (2009). Type I and Type II error concerns in fMRI research Rebalancing the scale. Social Cognitive \& Affective Neurosciences, 4, 423-428. doi:10.1093/scan/nsp052

Liu, Y., Liang, P., Duan, Y., Jia, X., Yu, C, Zhang, M.,... Li, K. (2011). Brain plasticity in relapsing-remitting multiple sclerosis: Evidence from resting-state fMRI. Journal of the Neurological Sciences, 304, 127-131. doi:10.1016/j.jns.2011.01.023

Maguire, E. A. (2001). Neuroimaging studies of autobiographical event memory. Philosophical Transactions of the Royal Society B, 356, 1441-1451. doi:10. 1098/rstb.2001.0944

Maguire, E. A., Kumaran, D., Hassabis, D., \& Kopel-man, M. D. (2010). Autobiographical memory in semantic dementia: A longitudinal fMRI study. Neuropsychologia, 48, 123-136. doi:10.1016/j.neur opsychologia.2009.08.020

Maguire, E. A., Vargha-Khadem, F., \& Mishkin, M. (2001). The effects of bilateral hippocampal damage on fMRI regional activations and interactions during memory retrieval. Brain, 124, 1156-1170. doi:10.10 93/brain/124.6.1156

Mainero, C, Caramia, F., Pozzilli, C, Pisani, A., Pestalozza, I., Borriello, G., ... Pantanob, P. (2004). fMRI evidence of brain reorganization during attention and memory tasks in multiple sclerosis. Neuro-Image, 21, 858 867. doi:10.1016/j.neuroimage.2003. 10.004

McDonald, W. I., Compston, A., Edan, G., Goodkin, D., Hartung, H.-P., Lublin, F. D., ... Wolinsky, J. S. (2001). Recommended diagnostic criteria for multiple sclerosis: Guidelines from the international panel on the diagnosis of multiple sclerosis. Annals of Neurology, 50(1), 121-127. doi:10.1002/ana.1032

Meulenbroek, O., Rijpkema, M., Kessels, R. P. C, Olde Rikkert, M. G. M., \& Fernandez, G. (2010). 
Autobiographical memory retrieval in patients with Alzheimer's disease. Neurolmage, 53(1), 331-340. doi:10.1016/j.neuroimage.2010.05.082

Montgomery, S. A., \& Asberg, M. (1979). A new depression scale designed to be sensitive to change. British Journal of Psychiatry, 134, 382-389. doi:10.11 92/bjp.134.4.382

Moran, J. M., Macrae, C. N., Heatherton, T. F., Wyland, C. L., \& Kelley, W. M. (2006). Neuroana-tomical evidence for distinct cognitive and affective components of self. Journal of Cognitive Neuros-ciences, 18, 5861594. doi:10.1162/jocn.2006.18.9.1586

Morgen, K., Sammer, G., Courtney, S. M., Wolters, T., Melchior, H., Blecker, C. R., ... Vaitlb, D. (2007). Distinct mechanisms of altered brain activation in patients with multiple sclerosis. Neurolmage, 37, 937-946. doi:10.1016/j.neuroimage.2007.05.045

Niendam, T. A., Laird, A. R, Ray, K. L., Dean, Y. M., Glahn, D. C, \& Carter, C. S. (2012). Meta-analytic evidence for a superordinate cognitive control network subserving diverse executive functions. Cognitive and Affective Behavioral Neurosciences, 12, 241-268. doi:10.3758/s13415-011-0083-5

Northoff, G., Heinzel, A., de Greek, M., Bermpohl, F., Dobrowolny, H., \& Panksepp, J. (2006). Self-referential processing in our brain-A meta-analysis of imaging studies on the self. Neurolmage, 31, 440-457. doi:10.1016/j.neuroimage.2005.12.002

Osterrieth, P. A. (1944). Le test de copie d'une figure complexe [Complex Figure Copy Test]. Archives de Psychologie, 30, 206-356.

Poettrich, K., Weiss, P. H., Werner, A., Lux, S., Donix, M., Gerber, J., ... Holthoff, V. A. (2009). Altered neural network supporting declarative long-term memory in mild cognitive impairment. Neurobiology of Aging, 30, 284-298. doi:10.1016/j.neurobiolaging. 2007.05.027

Rasmussen, A. S., \& Habermas, T. (2011). Factor structure of overall autobiographical memory usage: The directive, self and social functions revisited. Memory, 19, 597-605. doi:10.1080/09658211.2011. 592499

Raven, J. C. (1958). Advanced progressive matrices, set 1. Manual. London: H. K. Lewis.

Rey, A. (1941). L'examen psychologique dans les cas d'encéphalopathie traumatique [Psychological examination in cases of traumatic encephalopathy]. Archives de Psychologie, 28, 286-340.

Rey, A. (1964). L'Examen clinique en Neuropsycholo-gie [Clinical examination in neuropsychology]. Paris: PUF.

Shallice, T. (1982). Specific impairments of planning. Philosophical Transactions of the Royal Society B: Biological Sciences, 298, 199-209. doi:10.1098/rstb. 1982.0082

Shallice, T., \& Evans, M. E. (1978). The involvement of the frontal lobes in cognitive estimation. Cortex, 14 294-303. doi:10.1016/S0010-9452(78)80055-0

Sharot, T., Riccardi, A. M., Raio, C. M., \& Phelps, E. A. (2007). Neural mechanisms mediating optimism bias. Nature, 450, 102-105. doi:10.1038/nature06280

Sicotte, N. L, Kern, K. C, Giesser, B. S., Arshanapalli, A., Schultz, A., Montag, M., ... Bookheimer, S. Y. (2008). Regional hippocampal atrophy in multiple sclerosis. Brain, 131, 1134-1141. doi:10.1093/brain/ awn030

St Jacques, P. L, Kragel, P. A, \& Rubin, D. C. (2011). Dynamic neural networks supporting memory retrieval. Neurolmage, 57, 608-616. doi:10.1016/j.neu roimage.2011.04.039

Stroop, J. R. (1935). Studies of interference in serial verbal reactions. Journal of Experimental Psychology, 18, 643-662. doi:10.1037/h0054651

Summerfield, J. J., Hassabis, D., \& Maguire, E. A. (2009). Cortical midline involvement in autobiographical memory. Neurolmage, 44, 1188-1200. doi:10.1016/j. neuroimage.2008.09.033

Svoboda, E, McKinnon, M. C, \& Levine, B. (2006). The functional neuroanatomy of autobiographical memory: A meta-analysis. Neuropsychologia, 44, 2189-2208. doi:10.1016/j.neuropsychologia.2006. 05.023

Talairach, J., \& Tournoux, P. (1988). Co-planar stereo-tactic atlas of the human brain: 3-dimensional proportional system. Stuttgart: Georg Thieme.

Trapp, B. D., \& Nave, K. M. (2008). Multiple sclerosis: An immune or a neurodegenerative disorder? Annual Review of Neuroscience, 31, 247-269. doi:10.1146/ annurev.neuro.30.051606.094313 
Tulving, E. (2002). Episodic memory, from mind to brain. Annual Review of Psychology, 53(1), 1-25. doi:10.1146/annurev.psych.53.100901.135114

Vincent, J. L., Kahn, I., Snyder, A. Z., Raichle, M. E., \& Buckner, R. L. (2008). Evidence for a frontopar-ietal control system revealed by intrinsic functional connectivity. Journal of Neurophysiology, 100, 3328-3342. doi:10.1152/jn.90355.2008

Warrington, E., \& James, M. (1991). The Visual Object and Space Perception Battery (VOSP). Suffolk: Thames Valley Test Company.

Wechsler, D. (1997). Manual for the Wechsler Adult Intelligence Scale-Third Edition (WAIS-III). San Antonio, TX: The Psychological Corporation.

Williams, J. M. G., Ellis, N. C, Tyers, C, Healy, H., Rosen G., \& MacLeod, A. K. (1996). The specificity of autobiographical memory and imageability of the future. Memory and Cognition, 24(1), 116-125. doi:10.3758/BF03197278 